\title{
Primeiro Registro da Quenquém Cisco-da-Amazônia Acromyrmex hystrix (Latreille) (Formicidae: Myrmicinae) para o Estado do Maranhão, Brasil
}

\begin{abstract}
Wesley Dáttilo ${ }^{\circledR}$, Ricardo Eduardo Vicente ${ }^{2}$, Rafael Vieira Nunes $^{3} \&$ Marcela Soares Gigliotti Carvalho $^{3}$
1. Universidade Federal de Mato Grosso, Depto de Ecologia e Botânica, Lab. de Interação Inseto-Planta, e-mail: wdatillo@hotmail.com (Autor para correspondência ${ }^{\varpi}$ ). 2. Universidade Federal de Mato Grosso, Depto de Ecologia e Botânica, Lab. de Interação Inseto-Planta, e-mail: rickedu@bol.com.br. 3. Universidade Federal de Mato Grosso, Departamento de Biologia e Zoologia, e-mail: rafaelvnunes@yahoo.com.br, gigliotti marcela@yahoo.com.br.
\end{abstract}

\section{EntomoBrasilis 3 (3): 92-93 (2010)}

Resumo. Utilizando armadilhas pitfall, registra-se pela primeira vez quatro operárias da formiga quenquém cisco-da-Amazônia Acromyrmex hystrix (Latreille) (Formicidae: Myrmicinae) em uma caverna no estado do Maranhão, região nordeste do Brasil. Entretanto, não se pode inferir nenhuma informação adicional sobre a freqüência dessa espécie na região, uma vez que, o registro foi acidental. A região onde os indivíduos foram encontrados se encontra na divisa do Maranhão com Tocantins e sugere-se que essa espécie também esteja distribuída nesse estado.

Palavras-chave: Attini; caverna; distribuição geográfica; formiga; nordeste do Brasil

\section{First Record of the Quenquém cisco-da-Amazônia Acromyrmex hystrix (Latreille) (Formicidae: Myrmicinae) for Maranhão State, Brazil}

Abstract. It is recorded, for the first time, using pitfall traps, four workers of ant "quenquém-cisco-da-Amazônia", Acromyrmex hystrix (Latreille) (Formicidae: Myrmicinae) in a cave at state of Maranhão, northeastern Brazil. However, we could not imply any additional information about the abundance of this species in the region once that this record was accidental. The region where the individuals were found is placed at border of state of Maranhão and state of Tocantins suggesting that this species also occurs in this state.

Keywords: Ant; Attini; cave; geographic distribution; Northeastern Brazil

A s formigas cortadeiras do gênero Acromyrmex pertencem àfamília Formicidae, subfamília Myrmicinae etribo Attini. Essa tribo é caracterizada por serem as únicas formigas a cultivarem fungos simbiontes para obterem grande parte do seu alimento (Fowler et al. 1993). De acordo com MARICONI (1970) o gênero Acromyrmex é próprio da América do Sul e ocorre em quase todos os países do continente. Esse gênero é amplamente distribuído no território brasileiro, onde ocorrem 20 espécies e nove subespécies, sendo a maioria delas ainda pouco conhecidas, com exceção das poucas espécies consideradas potenciais pragas agrícolas (Della Lucia et al. 1993).

Acromyrmex hystrix (Latreillee) uma espécie com poucos dados da sua bioecologia na literatura, incluindo sua distribuição geográfica, e algumas poucas informações sobre hábitos de forrageamento e nidificação podem ser encontradas nos trabalhos de Weber $(1946,1947)$. Atualmente na América do Sul, essa espécie ocorre na Argentina, Brasil, Guiana, Guiana Francesa, Suriname, Venezuela (Weber 1946, 1947; Cantarelli 2005). Adicionalmente, FERnANdéz \& SEndoya (2004) relatam a ocorrência de $A$. hystrix para o Uruguai em uma lista de formigas neotropicais, entretanto, em levantamentos específicos da mirmecofauna deste país nunca foi registrado A. hystrix (Zolessi et al. 1989; Zolessi \& ABENANTE 1998).

No Brasil, de acordo com GonÇalves (1961) e KempF (1972) essa espécie é conhecida popularmente por "quenquém cisco-daAmazônia” e distribuída nos estados do Amazonas, Bahia, Goiás, Mato Grosso, Rondônia e Pará, entretanto, um estudo posterior ampliou sua distribuição para a Ilha de Santa Catarina no estado de Santa Catarina (Rosumek et al. 2008) (Figura 1).
Em coleta única realizada em caverna no mês de janeiro de 2010, na região do município de Estreito, sul do Estado Maranhão e divisa com o Estado de Tocantins (07037'11"S; $47^{\circ} 26^{\prime} 21$ ” $\mathrm{O}, 144 \mathrm{~m}$ ), foram coletadas quatro operárias de $A$. hystrix em armadilha pitfall. A caverna apresenta um desenvolvimento horizontal linear de 7,4 m e 0,9 $\mathrm{m}$ de altura, onde foram instaladas duas armadilhas. Todas as operárias foram coletadas somente na primeira armadilha, distante $1 \mathrm{~m}$ da entrada da caverna. A região onde a espécie foi encontrada situa-se em uma zona de transição entre os biomas Cerrado e Amazônia, na Bacia do rio Tocantins. A temperatura média anual é de $26,1^{\circ} \mathrm{C}$, com precipitação média anual de $1.718 \mathrm{~mm}$ e enquadrado em um regime de chuvas nitidamente tropical, onde o período chuvoso responde por mais de $80 \%$ do total anual precipitado (CESTE 2004). As formigas coletadas foram montadas em via seca e identificadas por meio de comparações feitas com a coleção disponível do Museu de Zoologia da Universidade de São Paulo (MZUSP). Todos os indivíduos coletados foram depositados na Coleção Entomológica da Universidade Federal de Mato Grosso (UFMT).

O município onde foram encontradas as operárias de A. hystrix situa-se na divisa entre os estados do Maranhão e Tocantins e, por esse motivo é provável que essa espécie também esteja distribuída no Tocantins. A metodologia utilizada neste trabalho não teve o intuito de coletar dados sobre abundância da espécie, por esse motivo, não se pode inferir nenhuma informação adicional sobre esse tipo de dado na região

Vale ressaltar que o registro foi feito por meio de coletas em caverna, e mesmo que $A$. hystrix não seja uma espécie estritamente cavernícola e sua coleta tenha sido acidental, na 


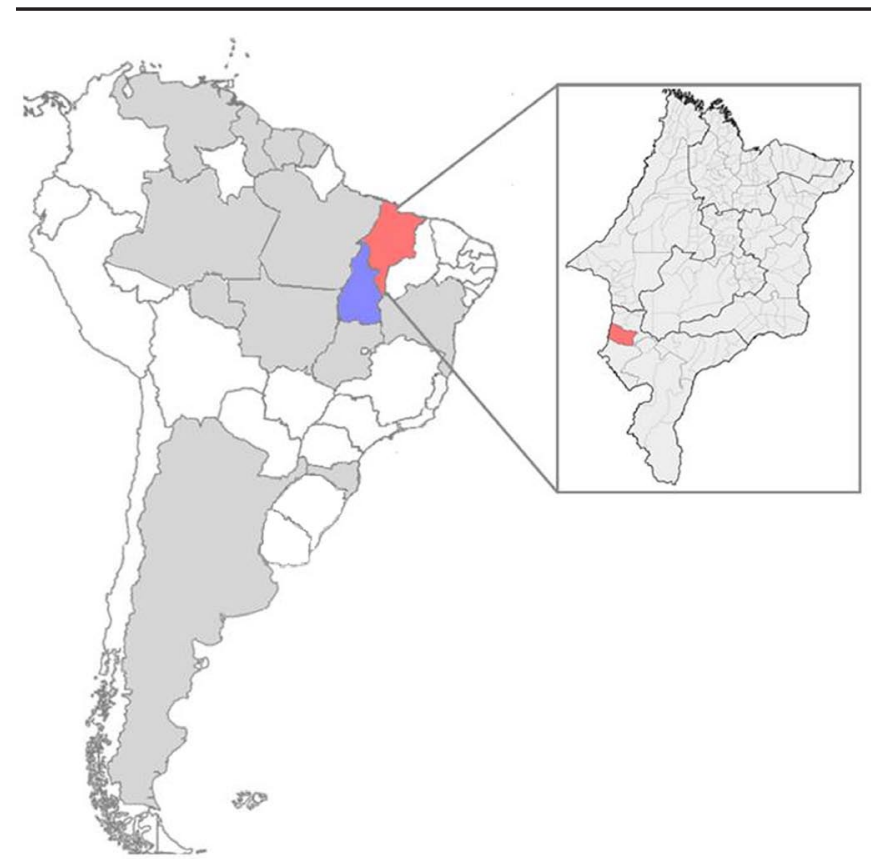

Figura 1. Distribuição atual da formiga Acromyrmex hystrix nos países da América do Sul e nos estados brasileiros (em Santa Catarina, a espécie foi coletada somente na Ilha de Santa Catarina). Observa-se em vermelho o novo registro para o estado do Maranhão (em detalhe o município de Estreito) e em azul sugere-se uma possível ocorrência para o estado de Tocantins.

maioria das vezes, as formigas associadas a este tipo de ambiente são consideradas animais troglóxenos, onde os ninhos podem ser estabelecidos no interior da caverna, mas as operárias devem retornar regularmente à superfície em busca de recursos alimentares (WEBER 1946, 1947; IzQUIERDO et al. 1986). Essa busca de recursos propicia a interação com diversos outros organismos, principalmente pelo fato de $A$. hystrix ser uma espécie de formiga cortadeira e necessitar de grande quantidade de material vegetal, não disponível no interior da caverna (DELla Lucia et al. 1993; DÁtTilo et al. 2009). Dessa forma, o presente registro aumenta a importância do conhecimento da fauna cavernícola do Brasil, que ainda é pouco conhecida (Trajano 1987; PinTO-DA-Rocha 1995).

Sugere-se para estudos futuros, trabalhos sobre a biologia e ecologia geral da espécie que ainda é pouco conhecida. Adicionalmente muitos estudos ainda devem ser feitos em regiões com baixa concentração de levantamentos faunísticos, onde muitas espécies consideradas raras ou ausentes possam estar presentes. Dessa forma, é possível que a espécie seja mais amplamente distribuída no país do que atualmente é considerado.

\section{AGRADECIMENTOS}

Os autores agradecem a Rodrigo Feitosa (MZUSP) pela confirmação na identificação da espécie de formiga. W. Dáttilo, R.V. Nunes, e R.E. Vicente agradecem ainda a CAPES pela concessão da bolsa de mestrado.

\section{REFERÊNCIAS}

Bolton, B., 1995. A taxonomic and zoogeographical census of the extant ant taxa (Hymenoptera: Formicidae). Journal of Natural History, 29: 1037-1056.

\section{Como citar este artigo:}

Dáttilo, W., R.E. Vicente, R.V. Nunes \& M.S. G. Carvalho, 2010. Primeiro registro da quenquém cisco-da-Amazônia Acromyrmex hystrix (Latreille) (Formicidae: Myrmicinae) para o estado do Maranhão, Brasil. EntomoBrasilis, 3(3): 92-93. www.periodico.ebras.bio.br/ojs
Cantarelli, E.B., 2005. Silvicultura de precisão no monitoramento e controle de formigas cortadeiras em plantios de Pinus. Tese de Doutorado em Engenharia Florestal - Centro de Ciências Rurais. Santa Maria-RS: Universidade Federal de Santa Maria, 95p.

CESTE, 2004. Estudo de Impacto Ambiental-Relatório de Impacto Ambiental do AHE Estreito. Rio de Janeiro: Ceste Consórcio Estreito de Energia, 235p.

Dáttilo, W., E.C. Marques, J.C.F. Falcão \& D.D.O. Moreira, 2009. Interações mutualísticas entre formigas e plantas. EntomoBrasilis, 2: 32-36.

Della Lucia, T.M.C., H.G. Fowler \& D.D.O. Moreira, 1993. Espécies de formigas cortadeiras no Brasil, p. 26-30. In: Della Lucia, T.M.C. (Ed). As formigas cortadeiras. Viçosa, Editora Folha de Viçosa, 252p.

Fowler, H.G., T.M.C. Della Lucia \& D.D.O. Moreira, 1993. Posição taxonômica das formigas cortadeiras, p. 4-30. In: Della Lucia, T.M.C. (Ed). As formigas cortadeiras. Viçosa, Editora Folha de Viçosa, 252p.

Mariconi, F.A., 1970. As saúvas. Agronômica Ceres, São Paulo, $167 \mathrm{p}$.

Fernández, F. \& S. Sendoya, 2004. Lista de las hormigas neotropicales. Biota Colombiana 5 (1): 3-109.

Gonçalves, C.R., 1961. O gênero Acromyrmex no Brasil. Studia Entomologica, 4(1): 113-180.

Izquierdo, I., A.L. Medina \& M. Díaz, 1986. La fauna invertebrada en las cuevas La Labrada y Las Mechas (Tenerife, Islas Canarias). Vieraea, 16: 309-320.

Kempf, W.W., 1972. Catálogo Abreviado das Formigas da Região Neotropical (Hymenoptera: Formicidae). Studia Entomologica, 15: 3-344.

Pinto-da-Rocha, R., 1995. Sinopse da fauna cavernícola do Brasil (1907-1994). Papéis avulsos de Zoologia, 39(6): 61-173.

Rosumek, F.B., M.A. Ulyssea, B.C. Lopes, J. Steiner \& A. Zillikens, 2008. Formigas de solo e de bromélias em uma área de Mata Atlântica, Ilha de Santa Catarina, sul do Brasil: levantamento de espécies e novos registros. Biotemas, 21 (4): 81-89.

Trajano, E., 1987. Fauna cavernícola brasileira: composição e caracterização preliminar. Revista Brasileira de Zoologia, 3(8): 533-561.

Weber, N.A., 1946. The biology of the fungus-growing ants. Part IX. The British Guiana Species. Revista de Entomologia, 17: 114-172.

Weber, N.A., 1947. Lower Orinoco River fungus-growing ants (Hymenoptera: Formicidae, Attini). Boletín de Entomología Venezolana, 6: 143-161.

Zolessi, L.C., Y.P. Abenante \& M.E. Philippi, 1989. Catalogo sistemático de las especies de Formicidos del Uruguay (Hymenoptera: Formicidae). Montevideo: Oficina Regional de Ciencia y Tecnologia de la Unesco para América Latina y el Caribe, 40p.

Zolessi, L.C. \& Y.P. benante, 1998. Las hormigas cortadoras del Uruguay (Hymenoptera: Formicidae). In: Formigas cortadeiras dos países do mercosul (E. Berti Fillo, F.A. Mariconi \& L.R. Fontes. Eds.), FEALQ, Piracicaba, Brasil, pp. 85-91.

Recebido em: 30/07/2010

Aceito em: 28/08/2010
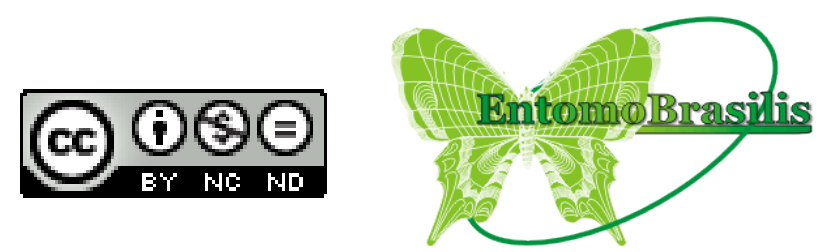